\title{
Pleural adenosine deaminase cut-off value for the diagnosis of tuberculous pleural effusion using the Diazyme assay
}

\author{
Hong Kong Med J 2018;24:207 \\ DOI: $10.12809 / \mathrm{hkmj} 187207$
}

To the Editor-We read with interest the article by Chang et al. ${ }^{1}$ In the diagnosis of tuberculous pleural effusion (TBPE), they established that $26.5 \mathrm{U} / \mathrm{L}$ is the optimal cut-off value for pleural fluid adenosine deaminase activity when using the Diazyme assay (Diazyme Laboratories, San Diego [CA], United States) on the UniCel DxC 800 Synchron Clinical System (Beckman Coulter, Brea [CA], United States). The Diazyme assay can be used on various analytical

(a)

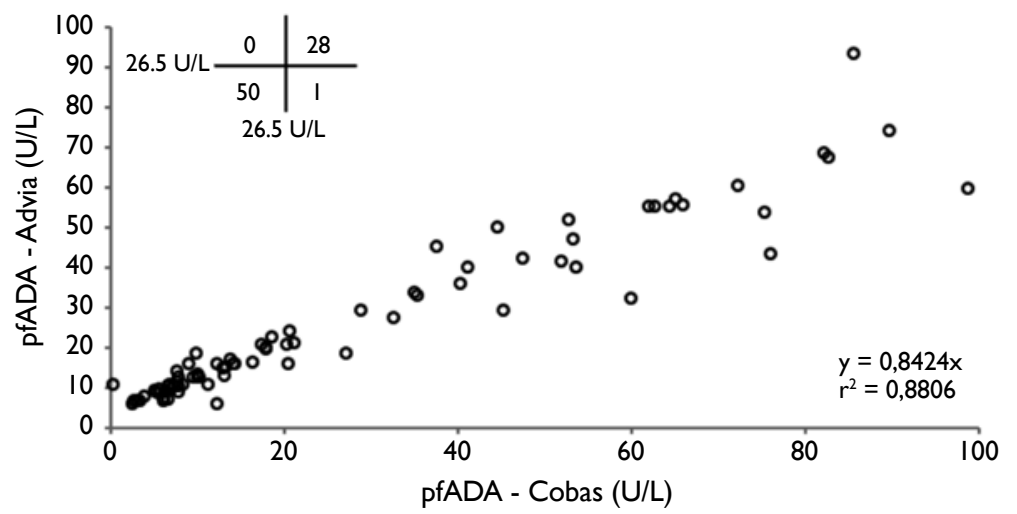

(b)

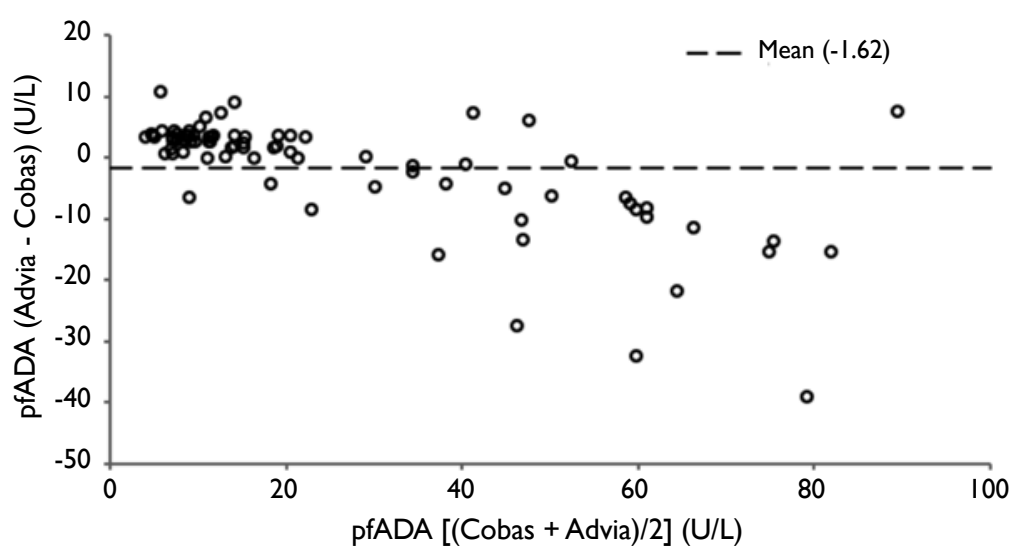

FIG. Comparison of Diazyme assay performance between Advia 1800 and Cobas 6000 systems

(a) Correlation plot showing that values obtained on the Advia 1800 correlated well with those obtained on the Cobas 6000; however, after use of the cut-off value proposed by Chang et al $(26.5 \mathrm{U} / \mathrm{L})$,' the interpretation of one sample was different (positive on the Cobas 6000 and negative on the Advia 1800). (b) Bland-Altman plot showing a negative bias ( -16\%), which increased with increasing pleural adenosine deaminase activity

Abbreviation: pfADA = pleural fluid adenosine deaminase systems. Therefore, one can ask the question: does the cut-off value differ according to the analytical system? The following two facts can provide an answer.

First, a cut-off value between 25 and $30 \mathrm{U} / \mathrm{L}$ has been previously recommended for TBPE diagnosis (ie, an interval including the cut-off value proposed by Chang et $\mathrm{al}^{1}$ ) if the Diazyme assay is used on the Cobas 6000 system (Roche Diagnostics, Meylan, France). ${ }^{2}$

Second, we have compared the results obtained from using the Diazyme assay on two analytical systems: Cobas 6000 (Roche Diagnostics) and Advia 1800 (Siemens Healthcare Diagnostics, Saint Denis, France) by parallel-testing 79 anonymous pleural fluid samples from different French hospitals. We observed a good correlation between the two analytical systems $\left(r^{2}=0.88 ; t\right.$ test for correlation coefficient, $\mathrm{P}<0.001)$, even though bias was observed ( 16\%). After using the cut-off value proposed by Chang et $\mathrm{al}^{1}{ }^{1}$ we observed only one misclassification between the two analytical systems (Fig).

In conclusion, we consider the cut-off value proposed by Chang et $\mathrm{al}^{1}$ for TBPE diagnosis to be appropriate on whichever analytical system the Diazyme assay is used.

\section{Declaration}

The authors have no conflicts of interest to disclose.

\author{
${ }^{1}$ Aurélie Servonnet, PharmD \\ ${ }^{1}$ Christine Frederic \\ ${ }^{1}$ Francisca Fargeau \\ ${ }^{2,3}$ Hervé Delacour *, PharmD \\ French Armed Forces Biomedical Research Institute, B.P. 73 - 91223 \\ Brétigny-sur-Orge Cedex, France \\ 2 Bégin Military Teaching Hospital, Fédération des Laboratoires, 69 \\ Avenue de Paris - 94163 Saint-Mandé Cedex, France \\ ${ }^{3}$ Val-de-Grâce Military School, 1 Place Alphonse Laveran - 75230 Paris \\ Cedex 05, France \\ *Corresponding author: herve.delacour@intradef.gouv.fr
}

\section{References}

1. Chang $\mathrm{KC}$, Chan MC, Leung WM, et al. Optimising the utility of pleural fluid adenosine deaminase for the diagnosis of adult tuberculous pleural effusion in Hong Kong. Hong Kong Med J 2018;24:38-47.

2. Delacour H, Sauvanet C, Ceppa F, Burnat P. Analytical performances of the Diazyme ADA assay on the Cobas ${ }^{\circ}$ 6000 system. Clin Biochem 2010;43:1468-71. 